\title{
New development of the Chinese bioanalytical landscape and update of the China Bioanalysis Forum (CFB)
}

\author{
Fan Jin*,1, Daniel Tang ${ }^{2}$, Kelly Dong ${ }^{3}$ \& Dafang Zhong ${ }^{4}$ \\ ${ }^{1}$ Bioanalytical Chemistry, Covance Pharmaceutical R\&D (Shanghai) Co., Ltd, Building \#3, No.3377 Kangxin.SIMZ, Pudong, \\ Shanghai 201318, PR China \\ ${ }^{2}$ Phanes Biopharmaceuticals, Songshan Lake Science and Technology Industry Park, Dongguan, Guangdong 523808, PR China \\ ${ }^{3}$ United-Power Pharma Tech Co., Ltd (UPP), 2F, Tower B, No. 33science Park Road, Changping District, Beijing 102206, PR China \\ ${ }^{4}$ Center for DMPK Research, Shanghai Institute of Materia Medica, Chinese Academy of Sciences, No. 501 Haike Rd, Zhangjiang \\ Hi Tech Park, Shanghai 201203, PR China \\ *Author for correspondence: Tel.: +86 18516755656; Fax: +86 2168078806; fan.jin@covance.com
}

"Regulatory reform is reshaping China's R\&D ecosystem."

First draft submitted: 11 January 2019; Accepted for publication: 21 February 2019; Published online: 4 April 2019

Keywords: China Bioanalysis Forum $(\mathrm{CBF}) \bullet$ China regulatory reform $\bullet$ drug innovation $\bullet$ regulated bioanalysis

China's drug innovation and development activity experienced rapid growth in the past decade, driven by huge unmet medical and patient needs, strong inflow of venture capital and a favorable state policy on innovation. China is establishing a regulatory environment with global standards for drug development. This article summarizes China's regulatory changes, plus provides an update on the China Bioanalysis Forum $(\mathrm{CBF})$ and progress on Regulated Bioanalysis in the country. The CBF has achieved its objectives in the past 6 years sharing technical knowledge and regulatory experience among the bioanalytical community. The CBF will continue its effort to promote scientific excellence, high quality and regulatory compliance in China and anticipates more close collaboration between itself and the international bioanalysis community.

\section{Drug innovation \& development in China}

China's drug innovation and development are moving forward rapidly. The Chinese drug market yielded pharma sales of $\$ 117$ billion in 2016, making China the second biggest pharmaceutical market in the world [1]. With increased government and private investment in pharma and biotech $\mathrm{R} \& \mathrm{D}$, a huge number of innovative compounds have entered into clinical development. Based on the BioCentury McKinsey report 2017, the combined value of all China biotech IPOs in the past 12 months reached $\$ 2.8$ billion, yielding a pipeline of 800 innovative molecules in preclinical to Phase III. In 2018, more than 350 New Chemical Entity INDs and about 150 Biologics INDs were submitted and more than 1500 clinical trials registered - more than double from the previous year [2].

In the past 10 years, immunotherapy such as checkpoint inhibitors is the most important breakthrough treatment for cancer. In June 2018, the China Food and Drug Administration (CFDA) approved the country's first cancer immunotherapy $\mathrm{OPDIVO}^{\circledR}$ from BMS for the treatment of locally advanced or metastatic non-small-cell lung cancer (NSCLC) [3]; in September 2018 CFDA approved Merck's KEYTRUDA ${ }^{\circledR}$ for treatment of melanoma. China's emerging biotechs and large pharma are catching up quickly in the immunotherapy front; most notable companies include Shanghai Junshi Biosciences, Innovent Biologics, Hengrui Pharmaceutical and BeiGene. State regulatory approved the first Chinese-made anti-PD-1 antibodies Junshi's Tuoyi for the treatment of melanoma and Innovent's Sintilimab for the treatment of Hodgkin's lymphoma in December 2018.

Another main driver for the rapid growth of new drug development in China is the regulatory reform. The International Council for Harmonization (ICH) met in Montreal, Canada in May 2017 and approved the CFDA as a new regulatory member. As a result, the CFDA has implemented reforms to align China's regulations with global standards. For instance, the CFDA is adopting 361 guidance documents from the ICH, FDA, EMA and 
WHO. We expect China will accelerate global development and the marketing process by following the same requirements.

The CFDA authorized the Center for Drug Evaluation (CDE) to grant final approval of IND application, supplement application and import license renewal. The CDE has significantly increased the number of reviewers to expedite review and approval timelines. In July 2018, the CFDA announced the launch of a 60-day clinical trial filing system that implemented priority review for innovative drug candidates and accelerated review and approval for selected medicines with high unmet medical need. The CFDA is mandating high quality data for drug development, Phase I and II clinical studies in China in parallel with global development. High quality data generated in China will support global filing. On 1 September 2018, the Chinese regulatory changed its name to the National Medical Product Administration marking a new era of local regulatory reform.

\section{Current status of bioanalysis in China}

Clinical success of New Chemical Entities and new biologics can only be achieved with an effective dosage regimen that has been designed with the understanding of pharmacokinetics (PK) and pharmacodynamics (PD) principles. Bioanalysis plays a critical role on TK/PK, biomarker and immunogenicity analysis throughout the drug development. With increased innovative drug discovery, as well as generic drug and biosimilar development, bioanalytical support is in high demand. China's regulatory changes have made a positive impact on regulated bioanalysis. Before July 2015, clinical sample analysis was supported mostly by hospitals or research institutes and some CROs; quality could vary substantially among different labs. On 22 July 2015, the CFDA mandated selfinspection and data verification of 1622 clinical studies pending CFDA review. Since then, high quality standards and routine inspection have become common. Pharmaceutical and biotechnology companies are more cautious in selecting bioanalytical labs [4]. In the meantime, clinical sample analysis is mostly performed in GLP/GCP compliance laboratories. In our opinion, the Chinese quality standard in bioanalysis is now of the international standard.

Currently, there are three main guidelines for Regulated Bioanalysis in China:

- CFDA Guidelines for Clinical Trial Bioanalytical Laboratory Management (Draft) Dec 2011 [5];

- China Pharmacopeia 2015, Guidance for Bioanalytical Method Validation [6];

- CFDA Announcement of Main Consideration Points for Clinical Data On-Site Verification, July 2015 [7].

The guidelines above follow the most updated guidance from the EMA and US FDA. However, no Chinese guidance has been implemented specifically on immunogenicity that addresses anti-drug antibody assay development and validation. In this regard, international guidance and global industry best practices are followed by most labs to support immunogenicity evaluation in China. Also new modalities such as gene and cell therapies require new technology platforms for PK and PD assays, in other words, qPCR and flow cytometry. These new biotherapeutics create opportunities and challenges for bioanalysis in terms of new technology platforms and regulatory compliance in China. With China regulatory reform, we anticipate more innovative drugs in clinical development. Therefore, we will have an opportunity to gain more experience in regulated bioanalysis through real projects.

\section{Development of China Bioanalysis Forum}

The China Bioanalysis Forum (CBF) was formed in September 2012. The steering committee includes seven members from academic, hospital and industry. The CBF Expert committee comprises 30 bioanalytical experts from China and overseas [8-11]. Six annual conferences were held from 2013 to 2018. Regional meetings were held in Beijing, Shanghai and Wuhan, which promoted knowledge and experience sharing at each part of the country. The 6th CBF annual conference took place in Wuhan, China in June 2018; approximately 500 professionals from pharmaceutical companies, academic institutes, CROs and hospitals participated in the meeting. Main topics included bioanalytical support throughout drug development, small and large molecule bioanalysis involving LCMS and LBA approaches, as well as bioanalysis for PK, biomarkers and immunogenicity. The most popular sessions were the Young Scientist Forum and Expert Panel Discussion.

The CBF has achieved its main objectives in the past 6 years, shared technical knowledge and regulatory experience among the bioanalytical community in China, and advocated scientific excellence, high quality and better regulatory compliance. The CBF promotes the harmonization of Chinese-regulated bioanalytical guidelines with international bioanalytical guidelines, supporting the execution of regulated bioanalysis in China based on the 
principle of international bioanalytical guidance and industrial best practice. In addition, the CBF encourages the exchange of academic and industrial research in the field of bioanalysis in China especially among young scientists.

The CBF will continue to enhance our collaboration with the international bioanalysis community such as EBF, AAPS and JBF, actively participate in the harmonization and globalization of international guidance and develop young scientists in the regulated bioanalysis field.

\section{Conclusion}

Regulatory reform is reshaping China's $\mathrm{R} \& \mathrm{D}$ ecosystem. China is establishing a regulatory environment with global standards for drug development. Since the CFDA issued the mandate of self-inspection and clinical data verification in July 2015, high quality standards and routine inspection have become a common practice. Regulated Bioanalysis in China has made steady progress in the past 3 years, with the adopted international standard. The CBF will continue its effort to promote scientific excellence, high quality and regulatory compliance in the bioanalytical community in China.

\section{Financial \& competing interests disclosure}

The authors have no relevant affiliations or financial involvement with any organization or entity with a financial interest in or financial conflict with the subject matter or materials discussed in the manuscript. This includes employment, consultancies, honoraria, stock ownership or options, expert testimony, grants or patents received or pending, or royalties.

No writing assistance was utilized in the production of this manuscript.

\section{References}

1. Mullard A. China Biopharma starts feeding global development pipeline. Nat. Rev. Drug Discov. 16(7), 443-446 (2017).

2. McKinsey \& Company: Building Bridges to Innovation. Shanghai (2017). www.bioindustry.org/uploads/assets/uploaded/62c081ea-59c4-4074-b763be4237988f04.pdf

3. Bristol-Meyers Squibb. China National Drug Administration approves country’s first immuno-oncology agent, Opdivo (nivolumab injection), for previously treated non-small cell lung cancer (NSCLC) (2018). https://news.bms.com/press-release/corporatefinancial-news/china-national-drug-administration-approves-countrys-first-imm

4. Dong K, Tang D. Bioanalysis outsourcing strategy in China. Bioanalysis 9(15), 1157-1159 (2017).

5. CFDA Guidelines for Clinical Trial Bioanalytical Laboratory Management (Interim) (2011). http://www.sda.gov.cn/WS01/CL0844/67395.html

6. 2015 China Pharmacopeia, Guidance for Bioanalytical Method Validation. http://www.drugfuture.com/standard/search.aspx

7. CFDA Announcement of Main Consideration Points for Clinical Data On-site Verification (2015). http://www.sda.gov.cn/WS01/CL0087/172936.html

8. Tang D, Zhong D, Dong K et al. Society spotlight: China Bioanalysis Forum. Bioanalysis 5(6), 641-43 (2013).

9. Tang D, Zhong D, Dong K. Conference Report for 2nd China Bioanalysis Forum Annual Meeting. Bioanalysis 6(20), 2709-2712 (2014).

10. Tang D, Zhong D, Dong K. The 3rd China Bioanalysis Forum annual meeting. Bioanalysis 7(22), 2853-2856 (2015).

11. Tang D, Zhong D, Dong K. The 4th China Bioanalysis Forum annual meeting. Bioanalysis 9(2), 159-162 (2016). 
